\title{
Virtual Smart Network Cell
}

\author{
Dr. Péter Kádár, senior member of IEEE
}

\author{
Óbuda University \\ Dept. of Power Systems, Alternative Energy Sources Knowledge Centre
}

Bécsi u. 94. Budapest H-1034 HUNGARY

Phone: +36 209447 241; fax: +30 1250 0940; e-mail: kadar.peter@kvk.uni-obuda.hu

\begin{abstract}
The local power generation facilities were interconnected into national, regional, continental and nowadays intercontinental networks. A lot of problems and tasks occur, like the controllability, the integration of the renewable sources, decrease of the spread over of the outages.

The present answers for the challenges are mainly extensive, the networks are enlarged, the traditional polluting generation capacities, the power plants, storages are developed and extended. Beside the "Electrical globalisation" occur local civil initiatives, the higher integration level of the grid resulted the need for new structures, as microgrids and locally controllable Smart Grids.

Our aim to develop structural and operational solutions for decrease the problems mentioned above, make the supply sustainable.

Beside the centralized intercontinental networks occur the microgrid, smart grid and "power quality island" solutions. Our basic concept is to develop a new virtual smart network cell haven't applied yet. It forms virtual microgrid island, uses the existing network, connected on IP base, aggregates small generators and consumers

Through this solution the load will be balanced, the dynamic of the operation will be decreased, the load-generation balance will be realized on local level, we move toward the sustainable power supply.
\end{abstract}

\section{Key words}

distributed generation, network structure,

\section{Background, problems to solve}

The local power generation facilities were interconnected into national, regional, continental and nowadays intercontinental networks. A lot of problems and tasks occur:

- Increasing level of aggregation, a lot of systems are connected

- The power systems are centrally organized (long term planning, operation, control, trade, etc.)

- Problem with the integration of Distributed Generation (DG) and RENewable energy sources (REN)

- For deregulation the development is declining, security decreases

- Actual trading interests contra long term balanced operation

- The integration of the REN sources lags behind the traditional generation modes
- Lack of the mobilisable resources

- The intensity of the network development and security decreases

- The short term trading interest wins over the long term stability

- The local energy supply is not developed (vs central generation)

- The sustainability is not secured

- The uncontrolled co-generation no makes flexible the network

- The uncontrolled wind parks makes difficult the load balance

- The un-forecasted cross border trading makes some overload

The present answers for the challenges are mainly extensive, the networks are enlarged, the traditional polluting generation capacities, the power plants, storages are developed and extended. Beside the "Electrical globalisation" occur local civil initiatives, the higher integration level of the grid resulted the need for new structures, as microgrids and locally controllable Smart Grids.

Seeking a best structural solutions one must take into account the following aspects too:

- network operation, failure analysis, forecast

- renewable sources

- power balance, scheduling

- network structures, topology

- optimization

- smart grids, etc.

\section{Existing structures}

In the frame if the traditional centralized energy supply there are more directions to build micro structures in the power systems. Up to this time geographically clearly limited structures were developed as microgrids, smart grids and power quality islands.

\section{A. Microgrids}

Isolated/Isolable, meshed network; Load and generation in the same measure; Only the ,unbalanced" load flows through the ,gate"; Minimal coordination; Medium 
voltage network; Possible internal tariffs; Developed internal coordination; Schedule handling; Developed IT (see fig. 1.).

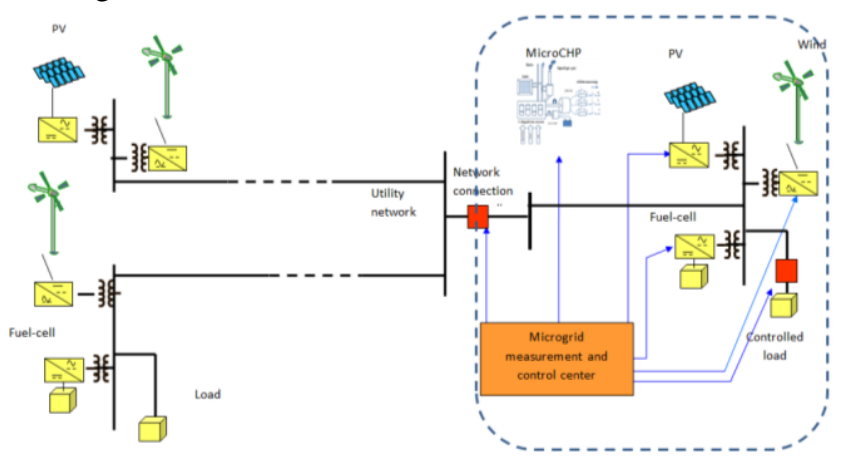

Fig. 1. The microgrid concept

\section{B. Partial smart grid}

Smart features are (by the San Diego Study [1]):

- $\quad$ automatically adapt protective systems to accommodate changing system conditions,

- re-route power flows, change load patterns, improve voltage profiles, and take other corrective steps within seconds of detecting a problem,

- $\quad$ enable loads and distributed resources to participate in operations,

- $\quad$ be inherently designed and operated with reliability and security as key factors, and

provide system operators with advanced visualization tools to enhance their ability to overview the system, etc.

The smart grid detects and addresses emerging problems on the system before they affect service; Respond to local and system-wide inputs and have much more information about broader system problems; Incorporate extensive measurements, rapid communications, centralized advanced; diagnostics, and feedback control that quickly return the system to a stable state after interruptions or disturbances; smart metering - Automatic Meter Reading; Demand side management (see fig. 2.)..

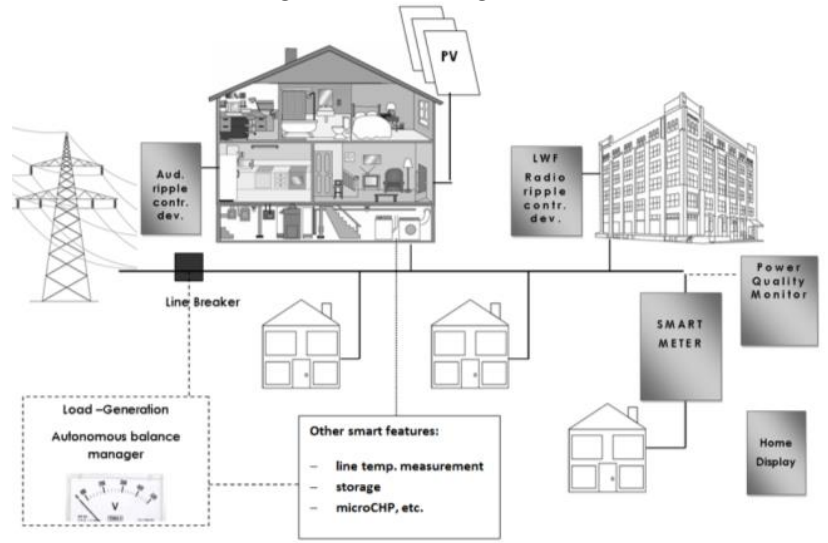

Fig. 2. The smart grid

\section{C. $\quad$ Smart quality island}

It isn't an isolated island but a network part connected by filters, impedances or FACT tools due to limitation of the harmful voltage, quality phenomena, power swings (see fig.3.).

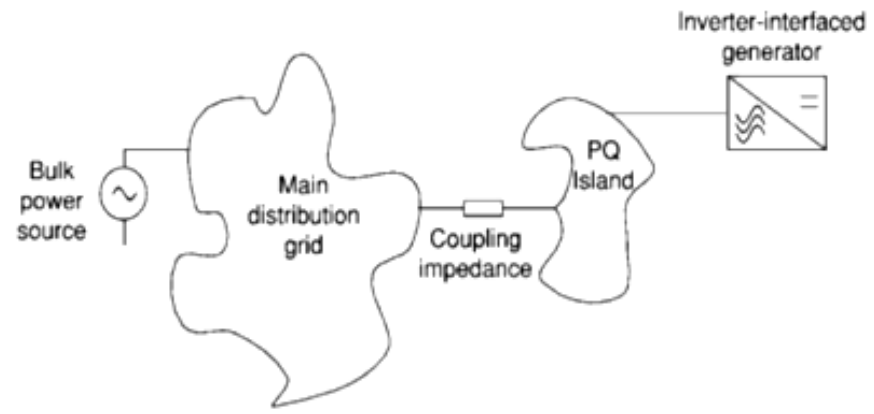

Fig. 2. The power quality island approach [2]

\section{The VPP}

The Virtual Power Plant (VPP) solutions expand over the limited area, virtually aggregate a bundle of the generators.

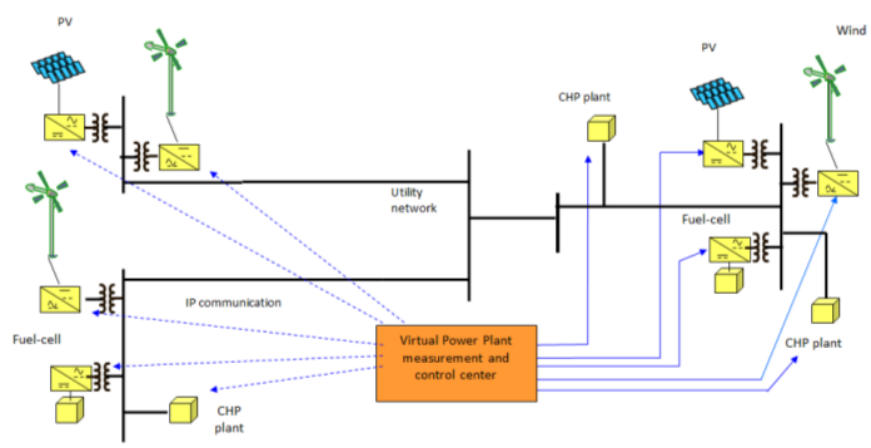

Fig. 3. VPP solution

\section{The virtualization}

The previous structures gives respectable steps toward the sustainable supply but

- The microgrids can be applied only in limited cases (e.g. in emergency), if we have a good network supply there is no why to isolate a part of the grid.

- Instead of greenfield investment smart grid construction the existing grids are extended more and more smart features.

- The power quality island is not typical in the general supply only in special industrial environment.

We recommend the virtual smart network cell (VSNC) haven't applied yet. The VSNC

- connected on IP base

- forms virtual microgrid island

- uses the existing network

- agree gate small generators and consumers

\section{A. VSNC structure}

The VSNC isn't isolated from the utility network, superposed on the large network, aggregate elements in large "electrical distance" in an ad-hoc bundle. The load and generation are in the same range, the low/medium voltage network is used (see fig.4.). 


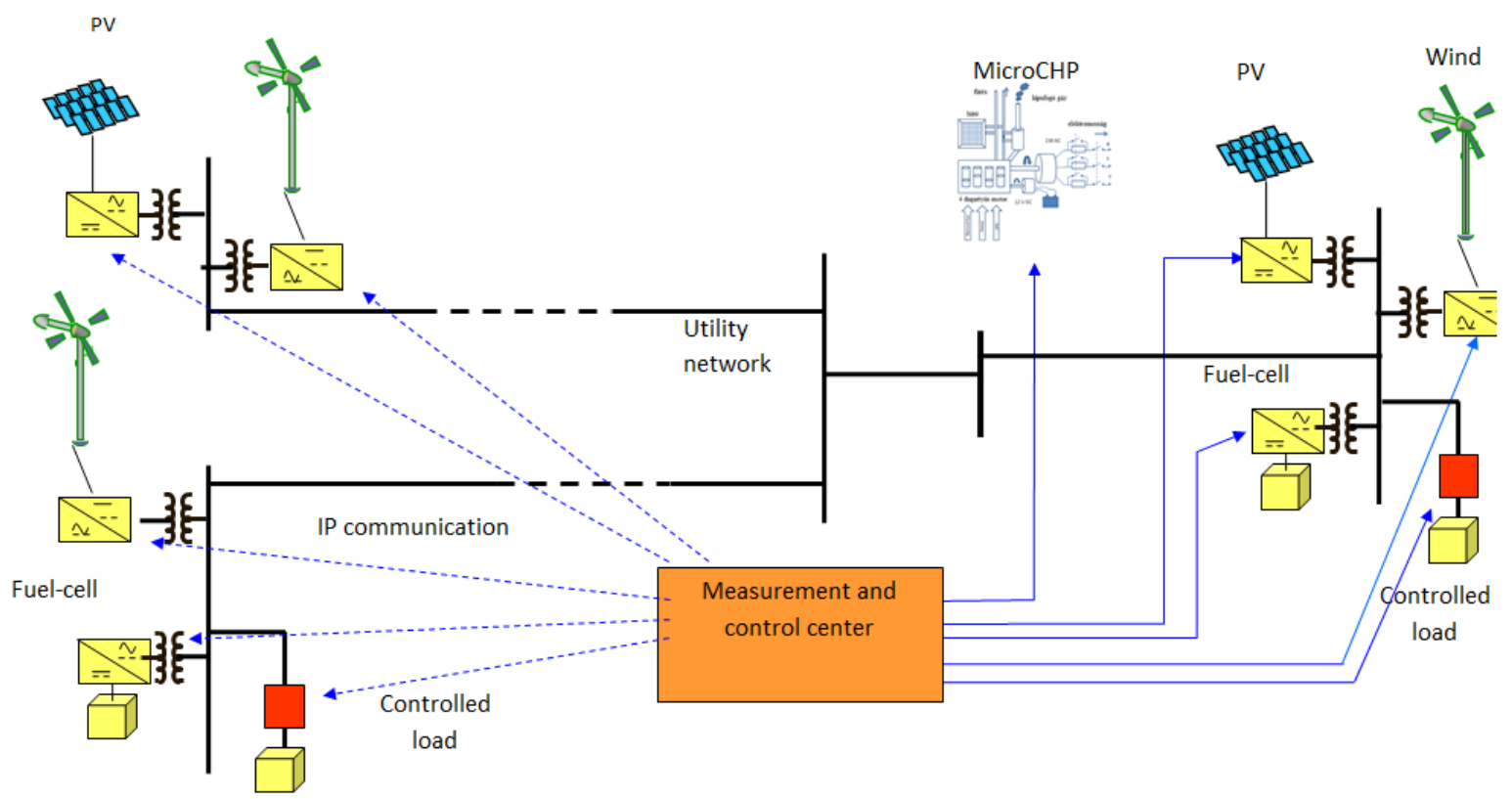

Fig. 4. The planned Virtual Smart Network Cell

The VSN Cells controls some elements of the network (of the county, of the country, of the region). One physical element of the of the whole network can be element of only one VSNC. The VSNC-s' areas are overlapping each-other (see fig.5.).

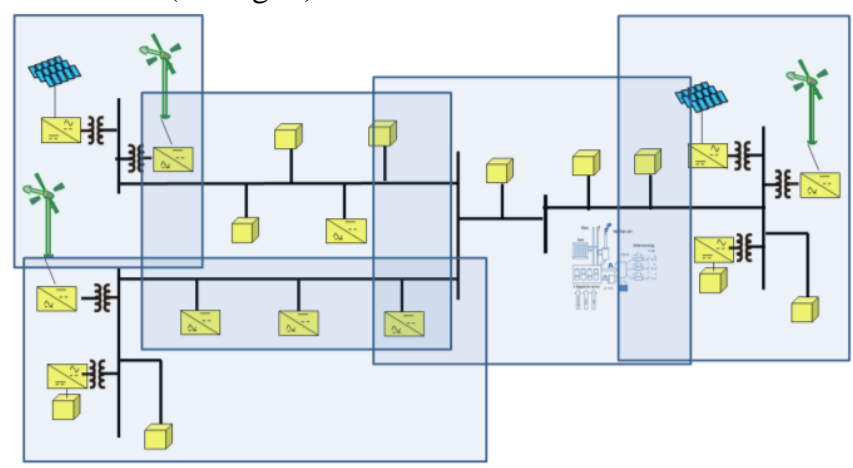

Fig. 5. Overlapping Virtual Smart Network Cells

\section{B. Element of VSNC}

The VSNC has intelligent elements, as generator, load and storages. Intelligence means that these elements identify its actual state, communicates it to the VSNC centre and receives commands from the VSNC centre.
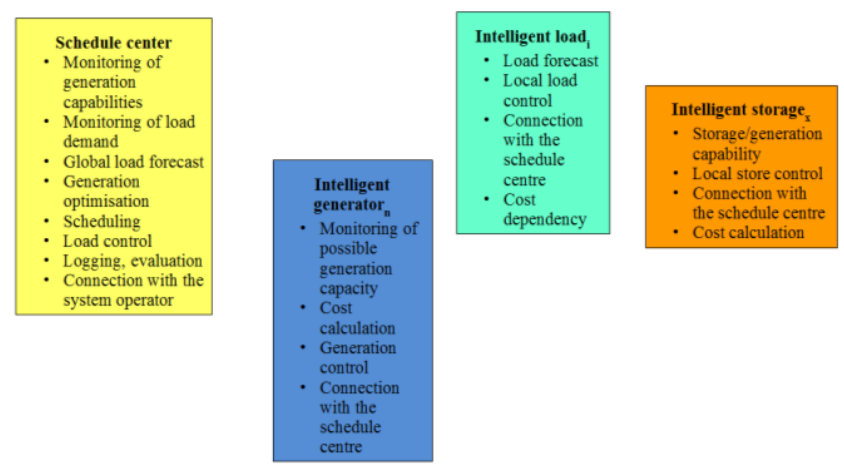

Fig. 6. Main elements of Virtual Smart Network Cell
The units provide information for the forecast (actual state, condition, capabilities). Also send some information to the centre for the cost calculations (see fig. 6.).

\section{Communication of VSNC}

The elements are connected by IP network. The IP net can be reached at almost all over the world, at $99 \%$ at the possible participants of the VSNC. The availability of the IP is about 99\%, so it is not applicable for remote control of nuclear plants. In our case the loss of information about small scale distributed generators doesn't cause trouble. Today these devices aren't connected in the VSNC cooperation, tomorrow large part of that will be. The penetration won't be $100 \%$. The security in the future must be raised by application of an internal security coded special protocol (see fig 7.)

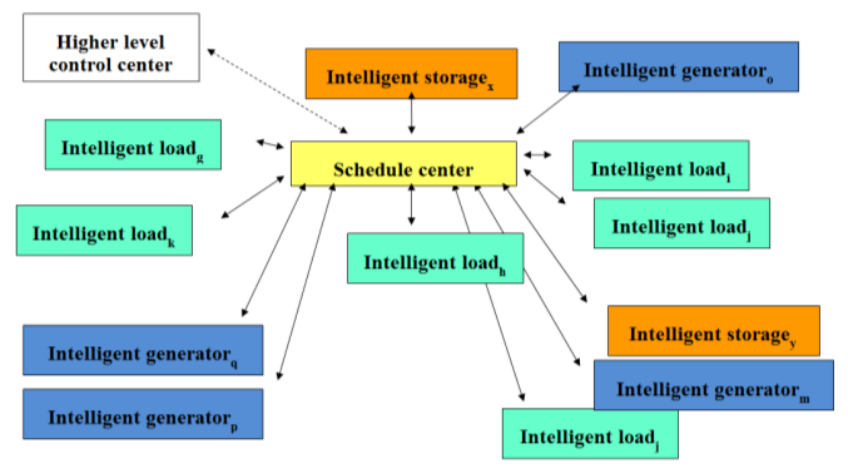

Fig. 7. Radial IP connections of the Virtual Smart Network Cell

\section{Operation of VSNC}

The controller

- sets the generation in the virtual net on the demand of the aggregated customers 
- $\quad$ on the base of previously defined schedule controls the load (demand side management)

- makes plan and forecasts

- $\quad$ keeps connection with the higher level network controller (system operator) due to the voltagereactive power control, loss minimization

The controller can have autonomous operation, without dispatcher surveillance. The control theoretically runs continuously on the power - practically in every 15 mins. Beside the online control the forecasts and schedule planning is also tasks of the controller.

On the VSNC level an aggregated schedule is formed:

$$
P_{V S N C}=\sum P_{\text {gen }}+\left(\sum P_{\text {vload }}+\sum P_{\text {vstore }}\right)
$$

where

$$
\begin{aligned}
& \mathrm{P}_{\mathrm{VSNC}}=\text { aggregated output of the VSNC } \\
& \mathrm{P}_{\text {gen }}=\text { generators in the VSNC }
\end{aligned}
$$

An advantage of the VSNC that reach all the small generators and the controllable load and controllable storages. The national control centre can't care about these numerous but small units. In this case these items must be subtracted from the national balance.

$$
\begin{aligned}
& \mathrm{P}_{\text {vload }}=(\text { controlled }) \text { loads of the VSNC } \\
& \mathrm{P}_{\text {vstore }}=\text { storages in the VSNC }
\end{aligned}
$$

The power system is controlled typically on national level. The Independent System Operator in the controlled area keeps the instantaneous power balance that comes from:

$$
\begin{aligned}
P_{\text {area }}=\sum P_{\text {Lgen }} & +\sum P_{V P P}+\sum P_{V S N C}+\sum P_{\text {Load }} \\
& +\sum P_{\text {store }} \\
& -\left(\sum P_{\text {vload }}+\sum P_{\text {vstore }}\right)
\end{aligned}
$$

where

$\mathrm{P}_{\text {area }}=$ instantaneous sum of the area

$\mathrm{P}_{\text {Lgen }}=$ large generators power in the area

$\mathrm{P}_{\mathrm{VPP}}=$ Virtual Power Plants

$\mathrm{P}_{\mathrm{Load}}=$ loads of the area

$\mathrm{P}_{\text {store }}=$ large storages in the area

In the present daily process the Transmission System Operator (TSO) collects the planned power schedules from the balance energy settling entities and the nationwide aggregated curve is forwarded to ENTSO-E confirmation (European Network of Transmission System Operators for Electricity). The process should be extended, the plans must be acquisited also from the VSNC-s - of course automatically.

\section{The demonstration model}

Although hundred types of data acquisition units and dozens of smart-style solution are recommended such supra-network virtual solution is not used yet. If large percentage of the small scale generator and loads should operate in thus structure the balancing work of the system operator would be easier.

We are building a demonstration system to evaluate the operation. We choose for data acquisition the National Instrument NI 6009 units (see fig.8.).

analog input e.g.

- actual power production of the unit (U, I, P)

- solar irradiation

- wind speed

- charge level of the battery

digital input

- availability

analog output

- allowed generation level (P,Q)

digital output

- turn off the device

- blocking signal

- remote start

- release/acknowledge, etc.

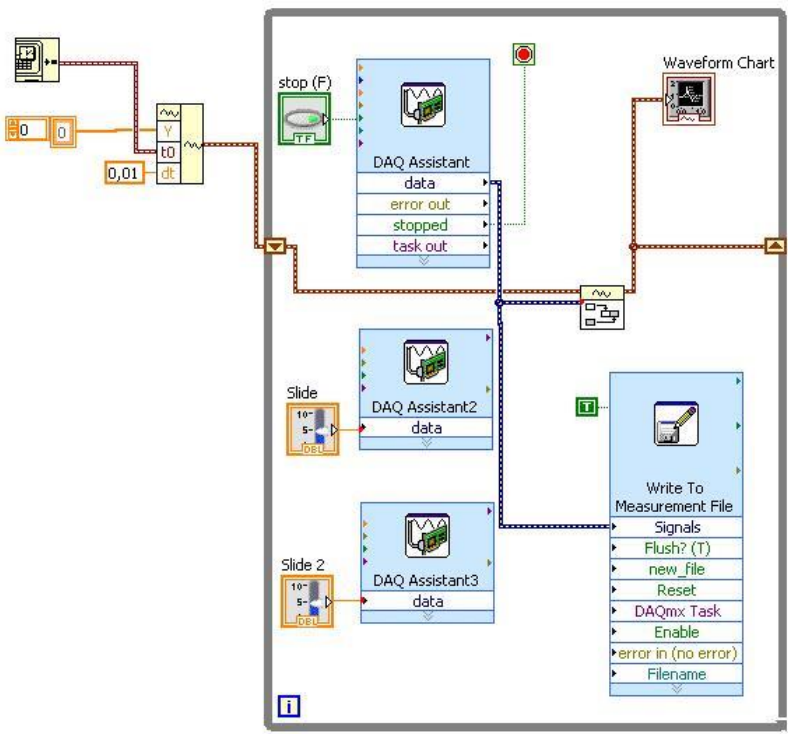

Fig. 8. Local data acquisition setting

The data are collected on local server close to the load/generation/storage devices. These servers are in IP connection with the central control unit. Theoretically the controller can be on other continent, but the recommended size of the cell is about

-1000 generator/load/storage unit

- 2-10 MW total power

- data exchange in every $15 \mathrm{~min}$

- units are connected to small/medium voltage system

- data exchange in every $15 \mathrm{~min}$

- geographic extent: $5-50 \mathrm{~km}^{2}$

The demonstration system is under construction in the campus of Óbuda University, in the Alternative Energy Sources Knowledge Centre. Here operate the following devices too:

- $\quad$ small- and medium scale PV systems (5 x $200 \mathrm{~W} ; 2$ x $3 \mathrm{~kW} ; 3$ × $28 \mathrm{~kW}$ )

- $\quad$ microCHP (cogeneration heat and power $-5 \mathrm{~kW}$ )

- $\quad$ small wind turbine $(0,5+1 \mathrm{~kW})$

- battery storage $(15 \mathrm{kWh})$ 
- $\quad$ EV charger $(3 \mathrm{~kW})$

- mini hydro $(0,3 \mathrm{~kW})$

- fuel-cell (20 W)

- heating device with storage capacities

(see fig. 9.)
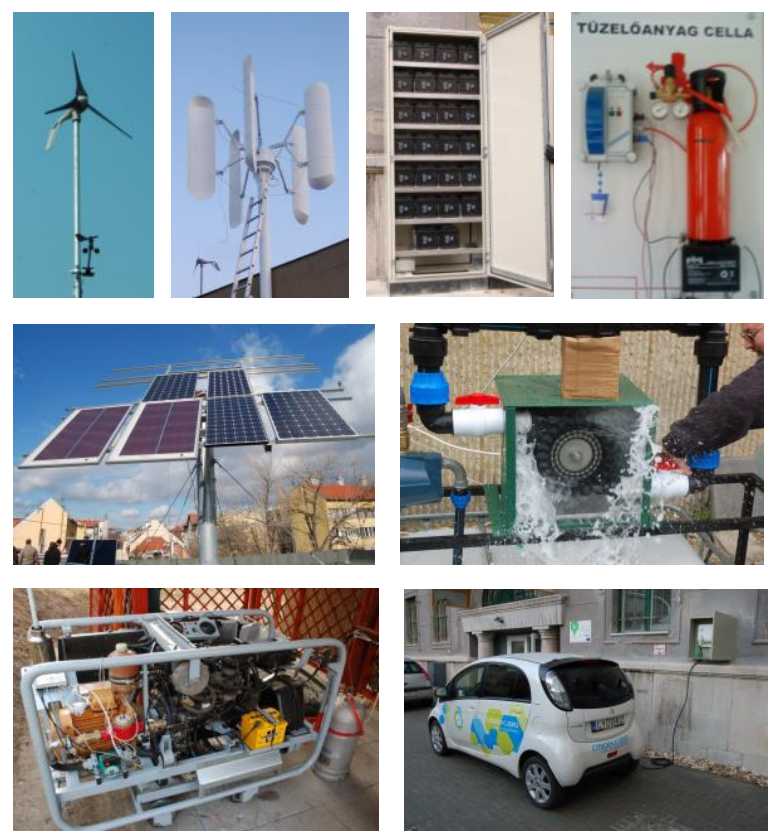

Fig. 9. Elements of the demonstration network

In the next figure the supposed daily power curve can be seen. The Virtual Smart Network Cell shows its aggregated curve for the higher network control levels.

\section{Daily course}

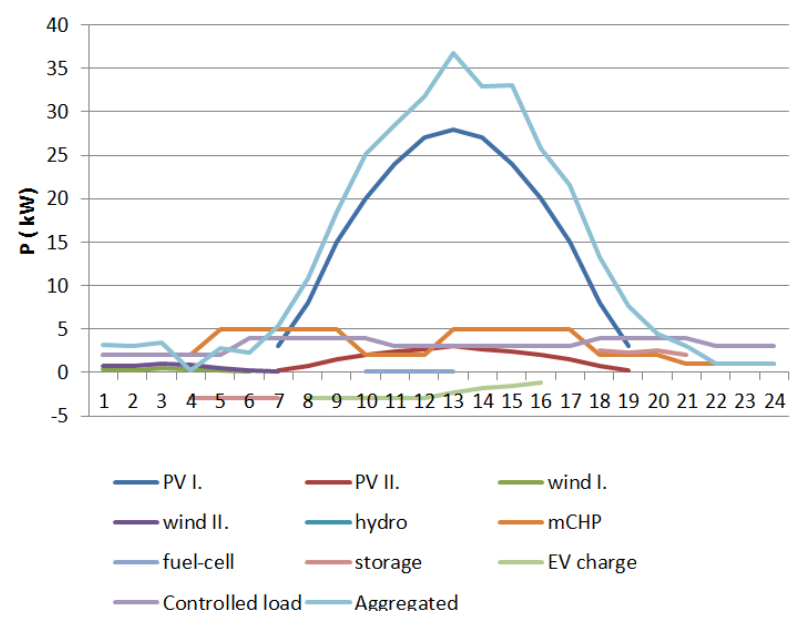

Fig. 10. Daily power course of the VSNC

\section{Conclusion}

Through the future distribution of the Virtual Smart Network Cell structures in the electrical power system we expect

- Growing reserves

- Integration of small weather dependent sources

- Better controllability

- Smaller dynamics

- Balanced load

- Transparency
- $\quad$ Easier settling

- Better planning

- Lower risk

The above mentioned solution supports the growths of the reliability of the power supply and decrease the pollution emission of the energy generation.

\section{References}

[1] https://lib.sandiego.edu/law/documents/centres/epic/061017 _SDSGStudyES_FINAL.pdf

[2] Tooraj Jamasb, William J. Nuttall, Michael G: Future electricity technologies and systems 2006 - Business \& Economics - p 426

[3] Hugo Morais - Péter Kádár - Marílio Cardoso - Zita A Vale: VPP Operating in the Isolated Grid, Proceedings of IEEE 2008 PES General Meeting, 20 - 24 July, 2008 in Pittsburgh, PA USA

[4] Peter Kadar: Performance measurements of car engine based MicroCHP test device; International Conference on Renewable Energies and Power Quality (ICREPQ'12); Santiago de Compostela (Spain), 28th to 30th March, 2012; Paper No.: 839

[5] Andrea Varga - Peter Kadar: PhotoVoltaic EV Charge Station; 12th IEEE International Symposium on Applied Machine Intelligence and Informatics (SAMI 2013) Herlany, Slovakia Jan.31- Feb. 2., 2013 pp 1-4

[6] Péter Kádár, Mark Karacsi: Stand alone island mode operation of microCHP device; EXPRES 2013, 22. March, 2013 Subotica, Serbia pp 1-5

[7] Péter Kádár - Mark Karacsi: Requirements of island mode controller for microCHP in micro grid, SISY 2013: IEEE 11th International Symposium on Intelligent Systems and Informatics; Subotica, Serbia, 2013.09.26-2013.09.28.

[8] Dr. Péter Kádár: Small scale balance manager for household energy system management; 8th IEEE International Symposium on Intelligent Systems and Informatics, September 10-11, 2010 Subotica, Serbia 